therapeutic protocol (after disease onset). The effect on disease severity (visual scores) as well as parameters of cartilage and bone destruction were evaluated.

Results Clinical scores were significantly reduced after IL-18 blockade (rhIL-18BP $1 \mathrm{mg} / \mathrm{kg}, \mathrm{p}<0.001, \mathrm{n}=13$; anti-IL18 IgG, $2 \mathrm{mg}, \mathrm{p}<0.05, \mathrm{n}=9$, Mann Whitney test, treated versus placebo groups). Histological examination showed cartilage protection (decrease erosion scores, $\mathrm{p}<0.05$ ) that was accompanied by significantly reduced levels of serum cartilage oligomeric matrix protein (an indicator of cartilage turnover) and VDIPEN expression (a neoepitope present after digestion by matrix metalloproteinases). X-ray analysis of joints provided evidence of reduced bone erosion.

Conclusion These results clearly demonstrate that neutralising endogenous IL-18 is therapeutically efficacious in the CIA model and support the use of IL-18 neutralisation as a novel cartilage and bone sparing therapy for the treatment of destructive arthritis. Recombinant hIL-18BP could therefore represent a new disease-modifying anti-rheumatic drug that warrants testing in clinical trials in patients with rheumatoid arthritis.

\section{OP0018 EARLY TREATMENT OF RECENT-ONSET RHEUMATOID ARTHRITIS PATIENTS IMPAIRS THE EFFECT OF HLA CLASS II ANTIGENS ON THE PROGRESSION OF JOINT DESTRUCTION}

${ }^{1} \mathrm{LR}$ Lard, ${ }^{1} \mathrm{JM}$ Hazes, ${ }^{1} \mathrm{FC}$ Breedveld, ${ }^{2} \mathrm{GM}$ Schreuder, ${ }^{2} \mathrm{RR}$ De Vries, ${ }^{2} \mathrm{E}$ Zanelli, ${ }^{1}$ TW Huizinga. ${ }^{1}$ Rheumatology; ${ }^{2}$ Immunohaematology and Bloodbank, Leiden University Medical Centre, Leiden, Netherlands

\subsection{6/annrheumdis-2001.943}

Background HLA class II antigens influence disease progression as measured by extend of joint destruction in RA. This effect is supposed to be caused by formation of auto reactive T-cells after presentation of (auto) antigens in the context of HLA class II.

Objectives To investigate whether in patients with recent-onset RA early DMARD treatment could prevent the involvement of autoreactive T cells, we analysed the association of HLA class II and joint damage in 110 patients with early RA that were treated according to the pyramid strategy with DMARDs and 98 patients with early RA that were promptly treated with DMARDs at two weeks after the first visit.

Methods DNA isolation, DRB1 typing and subtyping and DQB1 typing were performed. Extend of joint damage was measured by the modified Sharp score of the radiographs of hand and feet.

Results In the early treatment group the increase in the median Sharp score in the SE+ group was 1.0 to 5.0 in contrast to 1.0 to 3.0 in the SE- group ( $\mathrm{P}>0.5$ ). However, in the pyramid group the increase in joint damage was 0.0 to 16.0 in the $\mathrm{SE}+$ group in contrast to 0.0 to 5.0 in the SE- group ( $\mathrm{p}<0.005)$. On the other hand, in the RF+ group of the early treatment group the increase in joint damage was 1.0 to 6.5 in contrast to 1.0 to 1.0 in the RF- group ( $p<0.005$ ). In the pyramid group the increase in joint damage was 0.5 to 17.0 in the $\mathrm{RF}+$ in contrast to 0.0 to 3.0 in the RF- group ( $\mathrm{p}<0.005$ ).

Conclusion This study shows that early anti-rheumatic drug treatment abolishes the effect of HLA class II alleles on extend of joint damage in early RA patients.

\section{OP0019 ENDOGENOUS DNA AND ARTHRITIS}

V Collins, S Hajizadeh, A Tarkowski. Rheumatology, University of Goteborg, Goteborg, Sweden

\subsection{6/annrheumdis-2001.944}

Background Recent studies have shown that DNA, in addition to functioning as hereditary material, is able to activate the immune system. In particular, bacterial DNA displays in vivo and in vitro immunostimulatory properties. These properties have been attributed to certain unique properties of bacterial DNA including high content of unmethylated cytosines in $\mathrm{CpG}$ dinucleotides. Although eukaryotic nuclear DNA is not immunostimulatory, we have found that mitochondrial DNA (mtDNA), like bacterial DNA, can activate inflammation.

Objectives The aims of our studies were to assess the arthritogenicity in mice of endogenous mtDNA sequences and to assess a potential role in human inflammatory disease.

Methods Highly purified mtDNA as well as synthetically produced oligonucleotides were injected intra-articularly into murine knee joints. The development of arthritis was evaluated histopathologically. In addition, we analysed by PCR the synovial fluids (SF) of rheumatoid arthritis (RA) patients for the presence of free mtDNA.

Results Intra-articular injection of mtDNA and fragments of the mitochondrial genome containing unmethylated CpG sequences gave rise to arthritis in mice. This inflammation was mediated by macrophages and their products, such as TNF-alpha. In contrast, T/B lymphocytes or granulocytes did not participate in the onset or progression of the disease. The SF of 40/54 RA patients tested contained extracellular, PCR-amplifiable mtDNA.

Conclusion Our results indicate that endogenous mtDNA is proinflammatory and may participate in the induction and/or propagation of arthritis in man.

\section{OP0020 TUMOUR NECROSIS FACTOR ALPHA ACTS DIRECTLY ON OSTEOCLAST FORMATION BY SYNOVIAL MCROPHAGES AND MONOCYTES IN RHEUMATOID ARTHRITIS}

Y Fujikawa, O Kudo, H Taira, I Itonaga, T Torisu. Department of Orthopaedic Surgery, Oita Medical University, Oita-Gun, Japan

\subsection{6/annrheumdis-2001.945}

Background Rheumatoid arthritis (RA) is a chronic inflammatory disease of unknown aetiology that results in progressive destruction of bone and cartilage in affected joints. It has been reported that in mouse bone marrow cell cultures TNF-alpha directly induces osteoclast formation and IL-1alpha stimulates osteoclast activation, and that this osteoclast formation and activation is independent of osteoprotegerin ligand (OPGL) signalling system. $^{1,2}$

Objectives TNF-alpha and IL-1alpha considered master cytokines in the process of RA but little is known about the direct effects of these cytokines on development of human osteoclasts. This study aims to determine the role of TNF-alpha and IL-1alpha, on synovial macrophage- and monocyte-osteoclast differentiation in RA.

Methods Macrophages and monocytes isolated from RA patients were cultured with TNF-alpha and/or IL-1alpha on glass coverslips and dentine slices in the presence of M-CSF. Anti-TNFalpha, anti-IL-1alpha and osteoprotegerin (OPG) was added to this cultures. Cultures were assessed for cytochemical and functional evidence of osteoclast differentiation. 
Results The addition of TNF-alpha and M-CSF was sufficient to induce RA synovial macrophage- and monocyte-osteoclast differentiation in the absence of OPGL. This was evidenced by the formation of tartrate-resistant acid phosphatase (TRAP), vitronectin receptor, cathepsin K-positive multinucleated cells on glass coverslips and lacunar resorption pits on dentine slices. When IL-1alpha was added to this cultures together with TNF-alpha, the number of lacunar resorption pits were increased. It was found that anti-TNF-alpha inhibited osteoclast formation by RA synovial macrophages and monocytes and that although the number of TRAP-positive multinucleated cells was not changed, inhibition of pit formation was seen when anti-IL-1alpha was added to RA macrophage and monocyte cultures. OPG, however, showed no effect on osteoclast formation and activation induced by TNF-alpha and IL-1alpha.

Conclusion This study has shown that TNF-alpha induces RA synovial macrophage- and monocyte-osteoclast differentiation and that IL-1alpha activates osteoclasts to resorb bone. This osteoclast formation and activation is independent of OPGL signalling system. The new mechanism, inflammatory bone resorption, may represent an important role in the RA joint destruction and osteoporosis.

\section{REFERENCES}

1 Kobayashi $\mathrm{K}$, Takahashi $\mathrm{N}$, Jimi $\mathrm{E}$, et al. Tumor necrosis factor alpha stimulates osteoclast differentiation by a mechanism independent of the ODF/RANKL-RANK interaction. J Exp Med. 2000;191: 275-86

2 Azuma Y, Kaji K, Katogi R, Takeshita S, Kudo A. Tumor necrosis factor-alpha induces differentiation of and bone resorption by osteoclasts. I Biol Chem. 2000:275:4858-64

\section{OP0021 EFFECTS OF TNF-ALPHA, IL-1 BETA AND IL-15 ON THE PRODUCTION/EXPRESSION OF OPG AND RANKL BY SYNOVIAL FLUID MONONUCLEAR CELLS AND FIBROBLAST-LIKE SYNOVIOCYTES ISOLATED FROM PATIENTS WITH RHEUMATOID ARTHRITIS (RA)}

${ }^{1}$ M Zió3kowska, ${ }^{1} M S$ Kurowska, ${ }^{1} A$ Radzikowska, ${ }^{1} \mathrm{G}$ fuszzzykiewizz, ${ }^{2}$ W Dziewczopolski, ${ }^{3}$ J Kowalczewski, 'W Maslinski. 'Department of Pathophysiology and Immunology; ${ }^{2}$ Policlinic; ${ }^{3}$ Clinic of Orthopaedy, Institute of Rheumatology, Warsaw, Poland

\subsection{6/annrheumdis-2001.946}

Background The receptor activator of nuclear factor $\kappa \mathrm{B}$ (RANKL), its receptor RANK and osteoprotegerin (OPG) are key regulators of bone remodelling that influence immune functions as well. RANKL is responsible for the development and activation of osteoclasts. OPG, a soluble decoy receptor for RANKL, competes with RANK for RANKL binding. Proinflammatory cytokines (TNF- $\alpha$, IL-1b and IL-15) play critical role in the pathogenesis of RA and bone erosions.

Objectives To compare the effects of TNF- $\alpha$, IL- $1 b$ and IL-15 on the production/expression of OPG and RANKL by synovial fluids mononuclear cells (SFMC) and fibroblast-like synoviocytes (FLS) isolated from RA patients.

Methods SFMC and FLS were obtained from 8 RA patients. The isolated SFMC and FLS (cultured in vitro for 2-5 passages) were stimulated with recombinant human TNF- $\alpha$, IL-1b or/and IL-15. The concentrations of OPG in culture supernatants were measured by a specific ELISA and the expression of surface RANKL and OPG was tested using flow cytometry.

Results SFMC and FLS from RA patients spontaneously produced considerable levels of OPG. The production of OPG by FLS was strongly enhanced by TNF- $\alpha$ and IL-1b, but not by IL15. In contrast, the production of OPG by SFMC was only slightly enhanced in the presence of TNF- $\alpha$, IL-1b or IL-15. Interestingly, additive effects of IL-15 and IL-1b or IL-15 and TNF- $\alpha$ on OPG production by SFMC were observed. In addition to the soluble form, high levels of surface expressed OPG on $\mathrm{CD}_{3} 3^{+} / \mathrm{SFMC}$, but not on $\mathrm{CD}^{+} /$SFMC, was observed. The high expression of RANKL was found on CD $33^{+} /$SFMC, intermediate on $\mathrm{CD}^{+} / \mathrm{SFMC}$ and low on FLS. Tested cytokines modulate the expression of RANKL and OPG, in a complex, cell type and time dependent manner.

Conclusion TNF- $\alpha$, IL-1b and IL-15 affecting balance between RANKL and OPG influence inflammation and bone destruction in RA patients.

\section{OP0022 MOLECULAR PROFILE OF RHEUMATOID ARTHRITIS- SYNOVIAL FIBROBLASTS DURING PROLIFERATION}

${ }^{1} \mathrm{~K}$ Masuda, ${ }^{1} \mathrm{R}$ Masuda, ${ }^{1} \mathrm{M}$ Neidhart, ${ }^{1} \mathrm{CA}$ Seemayer, ${ }^{2} \mathrm{BR}$ Simmen, ${ }^{3} \mathrm{BA}$ Michel, ${ }^{4} \mathrm{U}$ MuellerLadner, ${ }^{1}$ RE Gay, 'S Gay. 'WHO Collaborating Center for Molecular Biology and Novel Therapeutic Strategies for Rheumatic Diseases, Department of Rheumatology; ${ }^{2}$ Upper Extremity and Handsurgery, Schulthess Clinic, Zurich, Switzerland; ${ }^{3}$ Department of Rheumatology, University Hospital Zurich, Zurich, Switzerland; ${ }^{4}$ Department of Internal Medicine I, University of Regensburg, Regensburg, Germany

\subsection{6/annrheumdis-2001.947}

Background Synovial fibroblasts (SF) play an important role in chronic inflammation and joint destruction in rheumatoid arthritis (RA). While many anti-apoptotic mechanisms in RA-SF have been demonstrated, various molecules promoting the proliferation of RA-SF were also believed to participate in the pathogenesis of RA.

Objectives The aim of this study was to investigate the expression profile of RA-SF during proliferation and to explore the molecular mechanisms of synovial proliferation in RA.

Methods Cultured RA-SF in passage 6 from one patient were used for cDNA subtraction and RT-PCR. Total RNA was extracted from two culture conditions, 4 days after replating (low-density cells; LD) and 14 days after replating (high-density cells; HD), respectively. One $\mu \mathrm{g}$ of total RNA from each culture was used for cDNA synthesis with the SMART PCR cDNA Synthesis Kit (CLONTECH), then suppression subtraction hybridization was performed with the PCR-Select cDNA Subtraction Kit (CLONTECH). Forward (LD-specific) and reverse (HD-specific) subtracted cDNA was subcloned into PCR-Script Amp SK (+) cloning vector (STRATAGENE). The nucleotide sequences of these clones were analysed by commercial sequencing (Microsynth), then BLAST searches (NCBI) were performed to identify each clone. mRNA expression of distinct clones in RA-SF were confirmed by RT-PCR with primers specific for each gene. The expression and distribution of distinct genes were examined on paraffin-embedded sections of synovial tissues from 7 patients with RA and 3 non-RA patients by in situ hybridization with digoxigenin-labelled RNA probes specific for each gene.

Results 44 clones were upregulated in LD cells and 44 clones were upregulated in HD cells, respectively. 46 of 88 clones were identical to the sequences that have been already identified and characterised, including the sequences of enzymes for metabolic pathways (i.e., aldose reductase, glutamine synthetase), cytoskeletal proteins (i.e., CDC10, EPLIN), proliferation-associated molecules (i.e., S100A4, NAP-1 related protein), matrix proteins (i.e., EFEMP1, versican), and ribosomal proteins. On the other hand, 29 clones were identical to cDNAs that have been already identified, but with unknown functions so far. 13 gene fragments did not show significant homology to any sequence in the GenBank 